\title{
A MODEL FOR THE SHELL OF HD 50138*
}

\author{
LÉO HOUZIAUX \\ Université de Mons, Département d'Astrophysique, Belgium \\ and \\ YVETTE ANDRILLAT \\ Observatoire de Haute-Provence, France
}

(Paper read by J. P. Swings)

\begin{abstract}
A model for the shell star HD 50138 is inferred from the observation of the continuous spectrum from 0.14 to $10 \mu$.
\end{abstract}

\section{The Observations}

HD 50138 was discovered to be a Be star by Humason in 1920. Merrill mentioned in 1931 the variability of its spectrum, which has been studied by Doazan (1965) and ourselves (1972). Further examples of this variability are shown in Figures 1, 2, and 3. The spectra (dispersion $39 \AA^{-1} \mathrm{~mm}^{-1}$ ) cover the region from $\mathrm{H} \alpha$ to $\mathrm{P} 12$ and show striking changes in line profiles and intensities over periods of $24 \mathrm{~h}$. The HD spectral type is B8e but, on the basis of its Balmer discontinuity, the star has been classified B5 IV-V by one of us (Houziaux, 1960). New measurements of the strength of the photospheric $\mathrm{He}$ I lines ( $\lambda 4471$ and $\lambda$ 4026) lead to an effective temperature of $12000^{\circ}$ for the central star. In order to resolve the apparent contradiction between the spectral types as given by these two criteria, we have gathered as much information as possible on the continuous spectrum. The $\mathrm{S} 2 / 68$ orbiting telescope (Boksenberg et al., 1973) provided absolute fluxes between $\lambda 1400$ and $\lambda 2500 \AA$. We observed the continuous spectrum photographically in the region $\lambda 3200$ $\lambda 5000$ (at $66 \AA \mathrm{mm}^{-1}$ ) and in the $\lambda 5500-\lambda 9200$ wavelength interval (at $230 \AA \mathrm{mm}^{-1}$ ). $U B V$ colors have been published by Haupt and Schroll (1974), while $R, I, J, K$, and $L$ magnitudes are given by Allen (1973). All these data are summarized in Figures 4 and 5. $V, R$, and $I$ magnitudes are in good agreement with our photometric measurements. The $B$ filter however indicates a flux $10 \%$ higher than our photographic determination.

\section{Interstellar Reddening}

The color indices $B-V=0.0$ and $U-B=-0.37$ indicate that the star is abnormally reddened; the $U-B$ excess is -0.35 with respect to normal B-type stars, which corresponds to a difference of 0.13 in the Balmer discontinuity. From the He I lines, a

\footnotetext{
* The observations have been carried out at the $193 \mathrm{~cm}$ and $120 \mathrm{~cm}$ telescopes of the Observatoire de Haute-Provence (CNRS).
} 


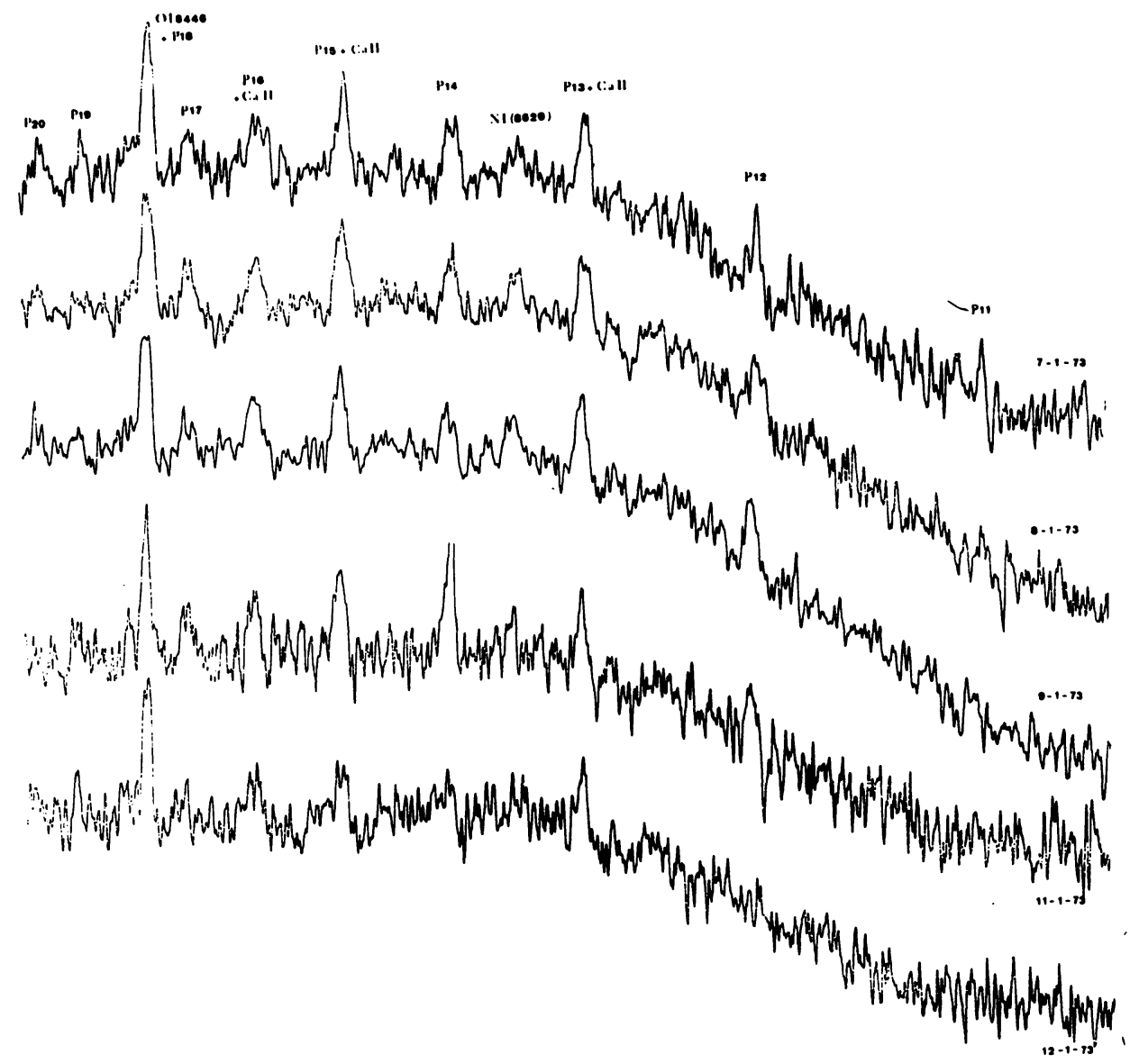

Fig. 1. Microphotometer tracings of the near infrared spectrum of HD 50138, showing rapid changes in the line profiles and intensities during the nights from Jan. 7 to Jan. 12, 1973.

temperature of $12000^{\circ}$ has been assigned to the stellar photosphere. Comparison of the far wings of $\mathrm{H} \gamma, \mathrm{H} \delta$ and $\mathrm{H} 8$ with computed profiles indicates a surface gravity of $10^{4} \mathrm{~cm} \mathrm{~s}^{-2}$. Hence, the star's photosphere can be classified B8 V. The normal $(B-V)_{0}$ color for such a spectral type is -0.12 . Hence, the color excess is 0.12 . If it is entirely due to interstellar reddening, it would correspond to an absorption of 0.8 . magnitude at $\lambda 2200 \AA$, according to Nandy (1974), if a normal $A_{V} / E(B-V)$ ratio is adopted. Such an absorption would imply the existence of a hump in the ultraviolet continuum around $\lambda 2200 \AA$, which is not observed (see Figure 4). On the other hand, an absorption $A_{V}=0.36$ locates the star at about $400 \mathrm{pc}$. Its absolute visual magnitude would then be -1.7 , in disagreement with the spectral type B8 V. In order to obtain an adequate absolute visual magnitude $(-0.1)$, the distance should be $200 \mathrm{pc}$, corresponding to an interstellar color excess $E(B-V)$ of 0.06 . 


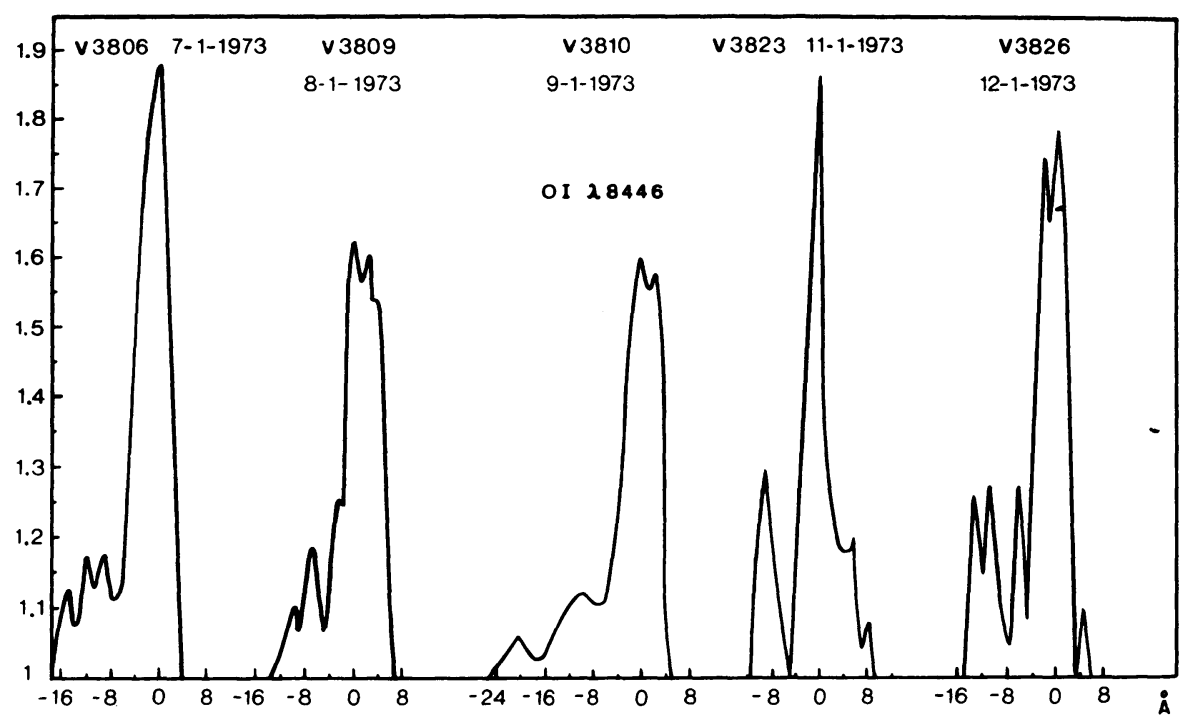

Fig. 2. Changes in the $O_{1}$ line at $\lambda 8446 \AA$.

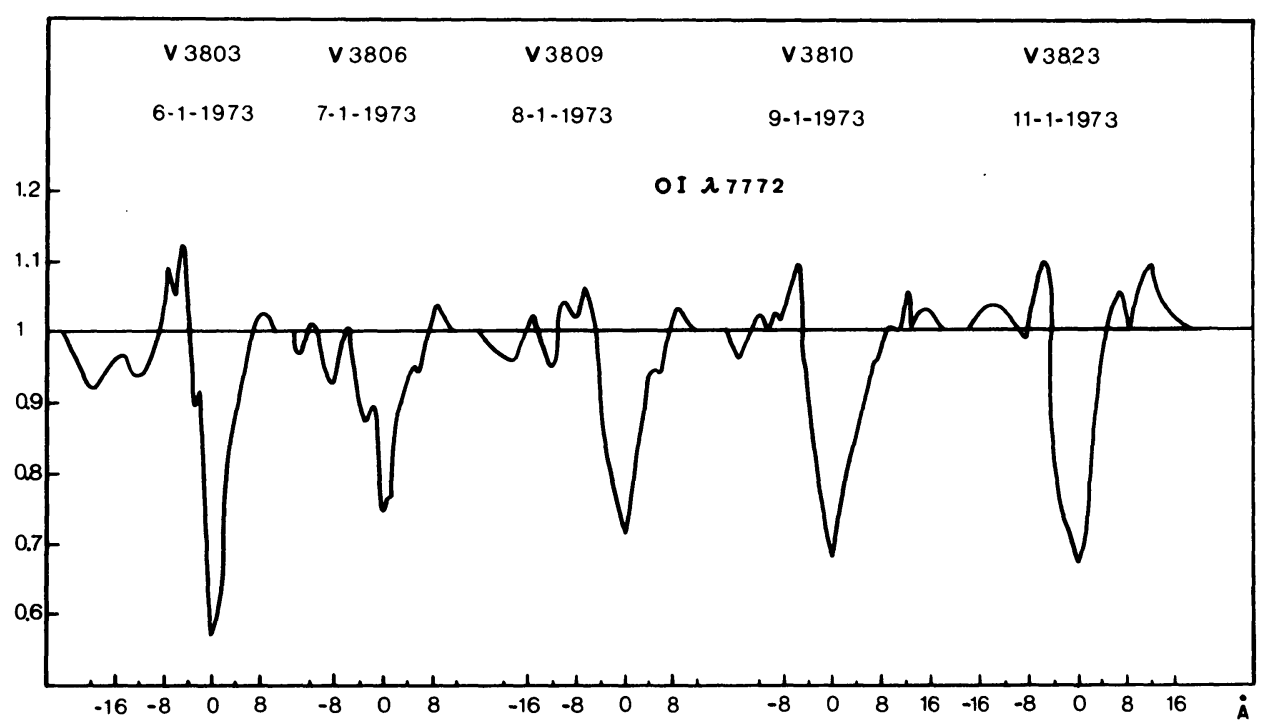

Fig. 3. Changes in the $\mathrm{O} I$ line at $\lambda 7772 \AA$.

\section{The Model}

Hence, the proposed model should account for:

(a) an 'intrinsic' color excess $E(B-V)=0.06$;

(b) a Balmer discontinuity smaller by 0.13 than the discontinuity assigned to a B8 V star; 


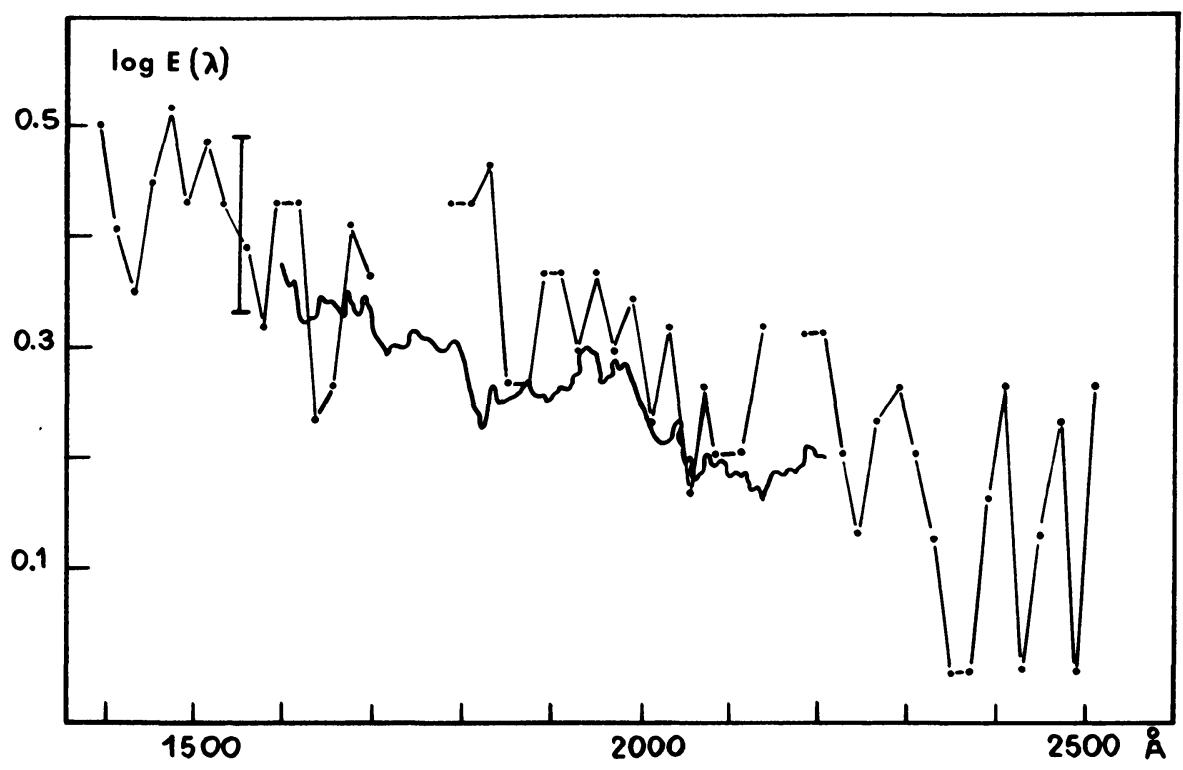

Fig. 4. Ultraviolet spectrum of HD 50138. Full line: computed spectrum. Dots: observed fluxes. See text.

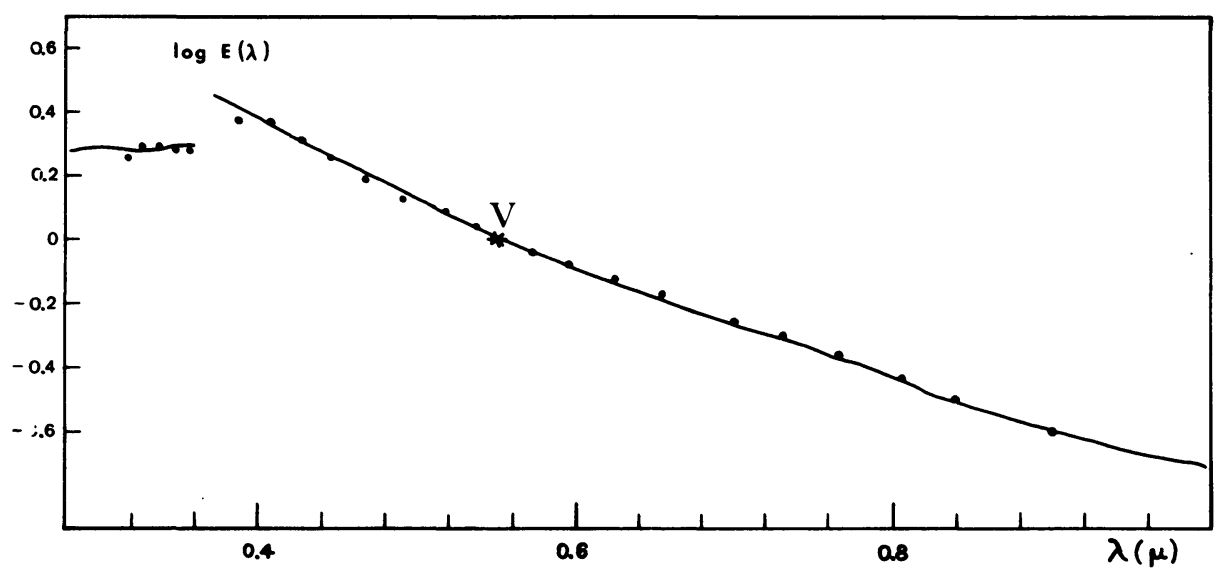

Fig. 5. Spectrum of HD 50138 in the region 0.3 to $1 \mu$. Full line: computed spectrum. Dots: observed fluxes. See text.

(c) the observed intensity of emission lines;

(d) the infrared excess;

(e) the presence of forbidden lines (namely $\mathrm{O}_{\mathrm{I}}$ ).

Starting with a B8 V central star with $T_{\text {eff }}=12000^{\circ}, \log g=4$, and $R=3.2 R_{\odot}$, we shall compute how the photospheric spectrum can be altered by a surrounding shell. Previous analysis of the shell absorption spectrum (Houziaux, 1960) led to an electron temperature of $10^{4 \circ}$ and an electron density of $3 \times 10^{11} \mathrm{~cm}^{-3}$. Because of the 
stellar rotation $\left(v \sin i=150 \mathrm{~km} \mathrm{~s}^{-1}\right)$, we shall admit that the shell is flattened with a flattening parameter $\alpha$ of 0.1 , although this value is by no means critical.

Assuming that the envelope is transparent to its own continuous radiation, the observed spectrum will result in the superposition of:

$$
\text { (central star radiation) } \cdot e^{-\tau}+\text { shell emission }+ \text { interst. abs. }
$$

The central emission is $4 \pi^{2} R_{*}^{2} F_{\lambda}$, where $F_{\lambda}$ is computed from a model atmosphere by Kurucz et al. (1974). The shell emission $E_{\text {shell }}(\lambda)$ consists of bound-free $\left(E_{b f}(\lambda)\right)$ and free-free emission $\left(E_{f f}(\lambda)\right)$ of a hydrogen plasma, which we assume to be fully ionized at the conditions prevailing in the shell. Using well-known notations, we shall write

$$
\begin{aligned}
E_{\text {shell }}(\lambda)= & \left(R_{\text {shell }}^{3} \alpha-R_{*}^{3}\right) \\
& \times\left[2.71 \times 10^{-21} N_{e}^{2} T^{-3 / 2} \sum_{n} \lambda^{2} n^{-3} 10^{68.544 / n^{2} T} e^{-1.439 / \lambda T} \bar{g}_{u}\right. \\
& \left.+8.54 \times 10^{-27} \lambda^{-2} N_{e}^{2} T^{-1 / 2} e^{-1.439 / \lambda T} \bar{g}\right]
\end{aligned}
$$

the sum being extended to appropriate $n$ quantum numbers, depending on the wavelength.

It is evident that $E_{\text {shell }}(\lambda)$ will show maxima at short wavelength edges of the hydrogen discontinuities, the maxima decreasing with increasing $n$. Precisely in our case, we note that there is a discrepancy of the Balmer discontinuity of 0.13 . In order to account for the 'filling-in' of the Balmer edge absorption, we determined from the above expressions $\boldsymbol{R}_{\text {shell }}$ to be $5 \boldsymbol{R}_{*}$, as all other parameters are known. If the star is seen edge-on (as may be inferred from the aspect of the shell absorption spectrum), we see that the opacity $\tau(\lambda)$ at the violet edge of the Balmer discontinuity is rather small:

$$
\tau(3650 \AA)=\tau_{b f}(3650 \AA)+\tau_{f f}(3650 \AA),
$$

where

$$
\begin{aligned}
& \tau_{b f}(3650 \AA) \sim\left(R_{\text {shell }}-R_{*}\right) 1.6 \times 10^{-17} N_{2} \\
& \tau_{f f}(3650 \AA) \sim\left(R_{\text {shell }}-R_{*}\right) 3.69 \times 10^{8}[1-\exp (-1.439 / T)] \bar{g} T^{-1 / 2} \nu^{-3} N_{e}^{2}
\end{aligned}
$$

$\mathrm{N}_{2}$ can be computed with the usual hydrogen recombination theory and is found to be $2 \times 10^{3} \mathrm{~cm}^{-3}$. The resulting value of $\tau(\lambda 3650 \AA)$ is 0.7 , which indicates that the opacity is indeed very low in the Paschen continuum.

\section{Comparison between Observed and Computed Fluxes}

The observed flux between $\lambda 1400 \AA$ and $1 \mu$ is given in Figures 4 and 5 . Satellite data are fairly uncertain, as indicated by the error bar. Absolute ultraviolet fluxes have been normalized to a $V$ magnitude 0.0 , assuming a $V=0.0, B-V=0$ star produces a flux of $3.64 \times 10^{-9} \mathrm{erg} \mathrm{cm}^{-2} \mathrm{~s}^{-1} \AA^{-1}$ at the effective wavelength of the $V$ filter at the top of the Earth's atmosphere. The dots in Figure 5 result from our 
photographic observations and from the photometric observations mentioned above. The normalization is the same as in Figure 4.

The computed fluxes $E(\lambda)$ are also reported on the same figures, with the same normalization:

$$
\log E(\lambda)=\log \left[4 \pi^{2} R_{*}^{2} F(\lambda) e^{-\tau_{\lambda}}+E_{\text {shell }}(\lambda)\right]-0.4 A(\lambda)+C,
$$

$C$ being a normalization factor and $A(\lambda)$ the interstellar absorption in magnitudes. Between 0.36 and $1 \mu$ the fluxes $F(\lambda)$ in the continuum have been computed in a straightforward manner from the above mentioned model. However, between $\lambda 1590$ and $\lambda 2200 \AA$, because of the extreme crowding of absorption lines, we have taken into account the effects of the lines, and folded the resulting fluxes with the instrumental profile of the S2/68 spectrometer (see Boksenberg et al., 1973). Several thousands of absorption lines have been considered, using a table established by Kurucz and Peytremann (1975). The interstellar extinction curve has been provided by Nandy (1974). It can be seen that the agreement is satisfactory throughout the spectrum, except between $\lambda 3700$ and $\lambda 4000 \AA$. This region is, however, crowded with many high Balmer lines.

\section{Hydrogen Shell Lines}

In order to check the validity of the model, we have computed the strength of the higher Paschen emission lines, where the opacity is very small. As P13, P15 and P16 are blended with the $\mathrm{Ca}$ II triplet, and P17 and P18 are seriously perturbed by the strong $O_{1}$ emission at $\lambda 8446 \AA$ (see Figure 1 ), we have used only P14.

The energy $E(14-3)$ emitted in the transition 14 to 3 may be written

$$
E(14-3)=\frac{4 \pi}{3}\left(R_{\text {shell }}^{3} \alpha-R_{*}^{3}\right) N_{14} A(14-3) h \nu_{14-3},
$$

where

$$
N_{14}=b_{14} N_{e}^{2} \frac{h^{3}}{\left(2 \pi m k T_{e}\right)^{3 / 2}} \frac{\varpi_{14}}{2} \exp \left(h R / 14^{2} T_{e}\right)
$$

with the usual notations. Taking $b_{14} \sim 1$ as a mean for the envelope, we find an equivalent width $W_{\lambda}$ of $4 \AA$ for P14, whereas we observe $3.2 \AA$. Taking into account the adopted approximations, this agreement may be considered as satisfactory.

On the other hand, the shell-is opaque to Balmer lines and, for the members of the Balmer series where no emission is detected, it is easy to separate the shell absorption component from the photospheric line. We have measured the lines from $\mathrm{H} 8$ to $\mathrm{H} 16$, and, using the isothermal thin layer approximation, as improved by Huang and Struve (1956), we find

$$
N_{2}\left(R_{\text {shell }}-R_{*}\right)=1.129 \times 10^{20} \frac{W_{\lambda}}{\lambda^{2} f}\left(\frac{R_{c}}{R_{c}-R_{0} / 2}\right),
$$

where $\boldsymbol{R}_{c}$ is the limiting depth $(0.8)$ and $\boldsymbol{R}_{0}$ the central depth of the shell line. Taking the value $R_{\text {shell }}-R_{*}$ as above, we find a mean value of $N_{2}=1.5 \times 10^{3} \mathrm{~cm}^{-3}$, in 
reasonable agreement with the value found in using the hydrogen recombination theory.

\section{Conclusions}

The proposed model is thus consistent with several observational facts:

(a) the B8 V central star accounts for the strength of the $\mathrm{He}_{\mathrm{I}}$ lines. It fits the observed ultraviolet flux in a spectral region very sensitive to the photospheric temperature;

(b) the shell reemission explains the apparently low value of the Balmer discontinuity $(0.27)$ for a $B 8 \mathrm{~V}$ star. It explains also a moderate $E(B-V)$ intrinsic reddening;

(c) the $\mathrm{H}_{\text {II }}$ shell model is compatible both with the strength of the Paschen emission lines and with the intensity of the Balmer absorption components.

It is clear however that the infrared excess mentioned by Allen (1973) must find its origin outside the $\mathrm{H}_{\mathrm{II}}$ shell. An $\mathrm{H}_{\mathrm{I}}$ region certainly exists around this $\mathrm{H}_{\mathrm{II}}$ shell, where low excitation forbidden lines may be formed. The infrared radiation may come from $\mathrm{H}^{-}$free-free and/or dust radiation in this region. So far, no evidence of molecular absorption has been detected in the spectrum of HD 50138; hence a binary nature for this object is not to be considered at present.

\section{Acknowledgements}

We would like to thank A. Delcroix (Mons) for computing the ultraviolet spectrum at our request. Thanks are due to the $\mathrm{S} 2 / 68$ reduction team in Liège for providing us with the ultraviolet spectral counts of HD 50138, and to E. Roques (Montpellier) and G. Houziaux (Mons) for help in the reduction of the various spectrometric data.

\section{References}

Allen, D. A.: 1973, Monthly Notices Roy. Astron. Soc. 161, 145.

Andrillat, Y. and Houziaux, L.: 1972, Astrophys. Space Sci. 18, 324.

Boksenberg, A., Evans, R. G., Fowler, R. G., Gardner, I. S. K., Houziaux, L., Humphries, C. M., Jamar, C., Macau, D., Malaise, D., Monfils, A., Nandy, K., Thompson, G. I., Wilson, R., and Wroe, H.: 1973, Monthly Notices Roy. Astron. Soc. 163, 391.

Doazan, V.: 1965, Ann. Astrophys. $28,1$.

Haupt, H. F. and Schroll, A.: 1974, Astron. Astrophys. Suppl. Ser. 15, 311.

Houziaux, L.: 1960, Publ. Obs. Haute Provence 5, No. 24.

Huang, S. S. and Struve, O.: 1956, Astrophys. J. 123, 231.

Kurucz, R. L., Peytremann, E., and Avrett, E. H.: 1974, Smithsonian Astrophysical Observatory, Washington D.C.

Kurucz, R. L. and Peytremann, E.: 1975, Special Report, Smithsonian Astrophysical Observatory.

Merrill, P. W.: 1931, Astrophys. J. 73, 348.

Nandy, K.: 1974, private communication. 